\title{
DYNAMICS OF THE PREDATOR-PREY MODELS ON THE TWO-PATCH FRAGMENTED HABITAT WITH DISPERSAL
}

\author{
KAIJEN CHENG, TING-HUI YANG AND JIN-WEI YU
}

\begin{abstract}
In this work, we consider the population-dispersal dynamics for predator-prey interactions in a two-patch environment. On each fragmented patch, there is a twospecies predator-prey ecological system. It is assumed that the predator species are mobile. The existence and local dynamics of boundary equilibria and interior equilibria with respect to parameters are completely classified. Moreover, global extinction results are established analytically. In particular, the phenomenon of over-exploitation is also found in these discrete patches models. Finally, some biological interpretations are discussed.
\end{abstract}

\section{Introduction}

Habitat fragmentation is an ecological phenomenon whereby discontinuities of habitat arise from naturally geological processes, or from human activity such as agriculture, rural development, urbanization and the creation of hydroelectric reservoirs [6, 9]. Habitat fragmentation is often a cause of species becoming threatened or endangered. So in order to preserve biodiversity. It is a matter of urgency to understand the effects of fragmented habitats $[7,8]$.

In this work, we investigate the dynamics of fragmented two-patch ecological systems. On each fragmented habitat, we assume a two-species food web model with Lotka-Volterra type interactions such that the predator species in the two-species system disperses between different patches with dispersal costs. More precisely, in the patch $i=1,2$, the following models are considered :

$$
\mathrm{P} i:\left\{\begin{array}{l}
\frac{d x_{i}}{d t}=r_{i} x_{i}\left(1-\frac{x_{i}}{K_{i}}\right)-a_{x y}^{i} x_{i} y_{i}, \\
\frac{d y_{i}}{d t}=\left(-d_{y_{i}}+a_{y x}^{i} x_{i}\right) y_{i}-\sum_{j \neq i} m_{i j} y_{i}+\sum_{j \neq i} m_{j i}\left(1-\varepsilon_{j i}\right) y_{j} .
\end{array}\right.
$$

Received July 20, 2015, accepted October 13, 2015.

2010 Mathematics Subject Classification. .

Key words and phrases. Predator-prey, two-patch habitat, dispersal costs, over-exploitation.

Corresponding author: Ting-Hui Yang.

Research supported in part by the NSC and NCTS of Taiwan. 
Here the constants $r_{i}, K_{i}$ and $d_{y_{i}}$ represent respectively the birth rate, environmental carrying capacity and the death rate for species $y$ on patch $i$. The parameter $0 \leq m_{i j}<1$ measures the dispersal rates between patches $i$ and $j$ with dispersal costs $0 \leq \varepsilon_{i j} \leq 1$. We make the following non-dimensional transformation,

$$
x_{i} \rightarrow x_{i} / K_{i}, \quad a_{y x}^{i} \rightarrow a_{y x}^{i} K_{i},
$$

retaining the other parameters in order to have the biological significance as rich as possible. Then system (1.1) can be rewritten in the forms,

$$
\begin{aligned}
& \text { P 1: }\left\{\begin{array}{l}
\frac{d x_{1}}{d t}=r_{1} x_{1}\left(1-x_{1}\right)-a_{x y} x_{1} y_{1}, \\
\frac{d y_{1}}{d t}=\left(-d_{y_{1}}+a_{y x} x_{1}\right) y_{1}-m_{12} y_{1}+m_{21}\left(1-\varepsilon_{21}\right) y_{2},
\end{array}\right. \\
& \text { P 2: }\left\{\begin{array}{l}
\frac{d x_{2}}{d t}=r_{2} x_{2}\left(1-x_{2}\right)-b_{x y} x_{2} y_{2}, \\
\frac{d y_{2}}{d t}=\left(-d_{y_{2}}+b_{y x} x_{2}\right) y_{2}-m_{21} y_{2}+m_{12}\left(1-\varepsilon_{12}\right) y_{1},
\end{array}\right.
\end{aligned}
$$

In two recent articles [1, 4], Lou and Wu [4] study a three-trophic level food chain model, proposed in [2], in two patches. The system consists of one resource species, two consumers, and a top predator. The top predator feeds on the two consumers and both consumers feed on the resource. Only the consumers move between the patches, possibly with a fraction of loss in population during the movement. If the two consumers have the same dispersal rate from patch 1 to patch 2, Lou and Wu show that the global dynamics of the model is completely determined by the standard Lyapunov function using LaSalle's invariance principle. They also show that there exists an "optimal" dispersal rate from patch 2 to patch 1 for the consumers, giving rise to a globally evolutionarily stable strategy and also a convergent stable strategy. Note that to generalize their results to more than 3 patches would not be an easy task since the effect of the geometric configuration of the patches comes into play.

In a related contribution Cressman and Křivan [1] consider the population-dispersal dynamics for two-species with predator-prey interactions or two competing species in a twopatch environment. They assume that both species (i.e., either predators and their prey, or the two competing species) are mobile and their dispersal between patches is directed to the higher fitness patch. Local stability of boundary and interior equilibria are established.

First, it is an interesting and important question to establish the global dynamics of (1.2)(1.3) rigorously. Such results would complement those in [1]. Understanding the global dynamics of two species on a fragmented habitat with two patches is an essential first step to understanding the results on more patches and with more species. Hence, in this work, we consider the models (1.2)-(1.3) in a two-patch environment. If the patches are isolated, that 
is $m_{12}=m_{21}=0$, then the local and global dynamics in each patch are well-known. Here we are interested in the effect of the dispersal between the two fragmented habitats on the dynamics of species and the meta-population [3] of the whole system, which is defined as the set of local populations which persists through a balance between local extinction and colonization.

For the predator-prey system on one patch with strong Allee effect in the prey, it is known that predator invasion leads to the extinction of both species. This phenomenon is called over-exploitation [10, 11, 12]. Mathematically, it means that a large enough initial predator population will always lead to the extinction of both species for any given initial prey population, i.e. convergence to the trivial equilibrium $(0,0)$. Although the systems $(1.2)-(1.3)$ have no Allee effect in the prey, in Proposition 2.9 we will show that over-exploitation arises due to the fragmented habitat.

The paper is organized as follows. In the next section we investigate a two-species predator-prey model in an environment with two patches analytically. First, results on positivity and boundedness of solutions are presented. In the special case where there is no dispersal from patch 1 to patch 2 , that is $m_{12}=0$, conditions for the existence of local stability of boundary and interior equilibria are presented. Criteria for global stability of the boundary equilibria are obtained by considering the limiting system as in Markus [5], using Lyapunov functions and LaSalle's invariance principle. Finally, numerical results on the global stability of the interior equilibrium are presented. In the final discussion section, we describe the ecological implications of our mathematical results and formulate our conclusions. We also point out some future research directions.

\section{Predator-prey systems in two patches}

In this section, first we show the positivity and boundedness of solution of (1.2)-(1.3). Then the existence and local stability of boundary and interior equilibria are established. Then classifications of all parameter corresponding to the global dynamics are presented. Finally, investigate analytically the dynamics of predator-prey system in the two patchy environments. If patches are isolated, that is $m_{12}=m_{21}=0$, then (1.2)-(1.3) are decoupled and each patch is a two-species predator-prey model which are well studied in the past decades. In term of notation, for the decoupled system of (1.2)-(1.3), it is obvious that

$$
\begin{aligned}
E_{0} & =(0,0,0,0), \\
E_{x_{1}} & =(1,0,0,0), \\
E_{x_{2}} & =(0,0,1,0), \\
E_{x_{1} y_{1}} & =\left(\frac{d_{y_{1}}}{a_{y x}}, \frac{r_{1}}{a_{x y}}\left(1-\frac{d_{y_{1}}}{a_{y x}}\right), 0,0\right) \text { if } d_{y_{1}}<a_{y x},
\end{aligned}
$$




$$
\begin{aligned}
E_{x_{2} y_{2}} & =\left(0,0, \frac{d_{y_{2}}}{b_{y x}}, \frac{r_{2}}{b_{x y}}\left(1-\frac{d_{y_{2}}}{b_{y x}}\right)\right) \text { if } d_{y_{2}}<b_{y x}, \\
E_{x_{1} x_{2}} & =(1,0,1,0), \\
E_{1} & =\left(\frac{d_{y_{1}}}{a_{y x}}, \frac{r_{1}}{a_{x y}}\left(1-\frac{d_{y_{1}}}{a_{y x}}\right), 1,0\right) \text { if } d_{y_{1}}<a_{y x}, \\
E_{2} & =\left(1,0, \frac{d_{y_{2}}}{b_{y x}}, \frac{r_{2}}{b_{x y}}\left(1-\frac{d_{y_{2}}}{b_{y x}}\right)\right) \text { if } d_{y_{2}}<b_{y x},
\end{aligned}
$$

are boundary equilibria. The only interior equilibrium is

$$
E_{*}=\left(\frac{d_{y_{1}}}{a_{y x}}, \frac{r_{1}}{a_{x y}}\left(1-\frac{d_{y_{1}}}{a_{y x}}\right), \frac{d_{y_{2}}}{b_{y x}}, \frac{r_{2}}{b_{x y}}\left(1-\frac{d_{y_{2}}}{b_{y x}}\right)\right) \text { if } d_{y_{1}}<a_{y x} \text { and } d_{y_{2}}<b_{y x} .
$$

Here we state some well-known classical results for $m_{12}=m_{21}=0$.

Proposition 2.1. Let the habitats patch 1 and 2 be isolated, that is, $m_{12}=m_{21}=0$. Then

(i) If $d_{y_{1}} \geq a_{y x}$, then $\lim _{t \rightarrow \infty} y_{1}(t)=0$. The boundary equilibrium $E_{x_{1}}$ is globally asymptotically stable (GAS) in the $x_{1}-y_{1}$ plane. Otherwise, if $d_{y_{1}}<a_{y x}$, then the equilibrium $E_{x_{1} y_{1}}$ is GAS in the $x_{1}-y_{1}$ plane.

(ii) Similarly, If $d_{y_{2}} \geq b_{y x}$, then $\lim _{t \rightarrow \infty} y_{2}(t)=0$. The boundary equilibrium $E_{x_{2}}$ is globally asymptotically stable (GAS) in the $x_{2}-y_{2}$ plane. Otherwise, if $d_{y_{2}}<b_{y x}$, then the equilibrium $E_{x_{2} y_{2}}$ is GAS in the $x_{2}-y_{2}$ plane.

Remark 2.2. By the previous results of proposition, in the decoupled system (1.2)-(1.3), equilibria $E_{x_{1} x_{2}}, E_{1}, E_{2}, E_{*}$ are GAS with positive initial condition in $\mathbb{R}^{4}$ if

$$
\begin{aligned}
& d_{y_{1}} \geq a_{y x} \text { and } d_{y_{2}} \geq b_{y x}, \\
& d_{y_{1}}<a_{y x} \text { and } d_{y_{2}} \geq b_{y x}, \\
& d_{y_{1}} \geq a_{y x} \text { and } d_{y_{2}}<b_{y x}, \\
& d_{y_{1}}<a_{y x} \text { and } d_{y_{2}}<b_{y x},
\end{aligned}
$$

respectively.

\subsection{Preliminary results and local stability of boundary equilibria}

To start our investigation of the coupled systems (1.2)-(1.3), we first show that solutions with positive initial conditions are bounded and positive for all positive time.

Lemma 2.3. Solutions of (1.2)-(1.3) are positive and bounded if they start with positive initial conditions. 
Proof. Consider the 4-dimensional phase space, $\left(x_{1}, y_{1}, x_{2}, y_{2}\right)$, it is obvious that systems (1.2)-(1.3) have invariant subspaces, $\left\{\left(0, y_{1}, x_{2}, y_{2}\right)\right\},\left\{\left(x_{1}, y_{1}, 0, y_{2}\right)\right\}$ and $\left\{\left(x_{1}, 0, x_{2}, 0\right)\right\}$ for all positive $x_{i}$ and $y_{i}$. In addition, on the hyperplane $\left\{\left(x_{1}, 0, x_{2}, y_{2}\right)\right\}\left(\left\{\left(x_{1}, y_{1}, x_{2}, 0\right)\right\}\right)$ with normal vector $(0,1,0,0)((0,0,0,1))$, the vector fields of systems $(1.2)-(1.3)$ point to non-negative cone of $\mathbb{R}^{4}$. Hence we show that non-negativity of all coordinates of solutions of (1.2)-(1.3) with positive initial conditions.

Now we show solutions of (1.2) - (1.3) are bounded. First, it is easy to see that limsup $\operatorname{su}_{t \rightarrow \infty}$ $x_{i}(t) \leq 1$ by the differential inequality. Moreover, define $D=\max \left\{\frac{a_{y x}}{a_{x y}}, \frac{b_{y x}}{b_{x y}}\right\}, \bar{D}=\min \left\{d_{y_{1}}, d_{y_{2}}\right\}$ and consider

$$
\begin{aligned}
\frac{d}{d t}\left(D \left(x_{1}(t)\right.\right. & \left.\left.+x_{2}(t)\right)+y_{1}(t)+y_{2}(t)\right) \\
& \leq D\left(r_{1} x_{1}\left(1-x_{1}\right)+r_{2} x_{2}\left(1-x_{2}\right)\right)-d_{y_{1}} y_{1}-d_{y_{2}} y_{2} \\
& \leq D\left(r_{1} x_{1}\left(1-x_{1}\right)+r_{2} x_{2}\left(1-x_{2}\right)\right)-\bar{D}\left(y_{1}+y_{2}\right) \\
& \leq M-\bar{D}\left(D\left(x_{1}+x_{2}\right)+y_{1}+y_{2}\right),
\end{aligned}
$$

where $M=D \sup _{t \geq 0}\left\{r_{1} x_{1}\left(1-x_{1}\right)+\bar{D} x_{1}+r_{2} x_{2}\left(1-x_{2}\right)+\bar{D} x_{2}\right\}$. Hence $D\left(x_{1}(t)+x_{2}(t)\right)+y_{1}(t)+$ $y_{2}(t)$ are bounded for all $t \geq 0$. And $x_{i}(t)$ and $y_{i}(t)$ are also bounded, because of non-negativity of $x_{i}(t)$ and $y_{i}(t)$. We complete the proof.

In order to couple the systems (1.2) and (1.3), we assume that one of the dispersal rates $m_{12}$ and $m_{21}$ is positive. Moreover, to simply the arguments we assume further another dispersal rate is zero. Hence, without loss of generality, we have the assumption,

(A1) $m_{21}>0$ and $m_{12}=0$,

throughout this work. So we obtain a simplified systems of (1.2)-(1.3) :

$$
\begin{aligned}
& \text { P 1: }\left\{\begin{array}{l}
\frac{d x_{1}}{d t}=r_{1} x_{1}\left(1-x_{1}\right)-a_{x y} x_{1} y_{1}, \\
\frac{d y_{1}}{d t}=\left(-d_{y_{1}}+a_{y x} x_{1}\right) y_{1}+m_{21}\left(1-\varepsilon_{21}\right) y_{2},
\end{array}\right. \\
& \text { P 2: }\left\{\begin{array}{l}
\frac{d x_{2}}{d t}=r_{2} x_{2}\left(1-x_{2}\right)-b_{x y} x_{2} y_{2}, \\
\frac{d y_{2}}{d t}=\left(-d_{y_{2}}+b_{y x} x_{2}\right) y_{2}-m_{21} y_{2} .
\end{array}\right.
\end{aligned}
$$

It is easy to see that $E_{x_{1} x_{2}}$ and $E_{1}$ are still boundary equilibria of (2.1)-(2.2). But $E_{2}$ is not an equilibrium anymore. In the following, we try to find all equilibria with positive $x_{2}$ and $y_{2}$ coordinates.

By the second equation of (2.2), it easy to to solve $x_{2}^{*}=\frac{d_{y_{2}}+m_{21}}{b_{y x}}$ and $y_{2}^{*}=\frac{r_{2}}{b_{x y}}\left(1-x_{2}^{*}\right)$ if $b_{y x}>d_{y_{2}}+m_{21}$. By system (2.1), we should solve the equations,

$$
\left(r_{1}\left(1-x_{1}\right)-a_{x y} y_{1}\right) x_{1}=0,
$$




$$
\left(d_{y_{1}}-a_{y x} x_{1}\right) y_{1}=m_{21}\left(1-\varepsilon_{21}\right) y_{2}^{*},
$$

to find equilibria. If $x_{1}^{*}=0$, then we have $y_{1}^{*}=\frac{m_{21}\left(1-\varepsilon_{21}\right)}{d_{y_{1}}} y_{2}^{*}$. So here is a boundary equilibrium

$$
\bar{E}_{2}=\left(0, \frac{m_{21}\left(1-\varepsilon_{21}\right)}{d_{y_{1}}} y_{2}^{*}, x_{2}^{*}, y_{2}^{*}\right) .
$$

Otherwise, if $x_{1}^{*}>0$, then the positive equilibrium $\bar{E}^{*}$ exists if and only if we can find a positive number $x_{1}^{*}$ such that $x_{1}^{*}<\min \left\{1, \frac{d_{y_{1}}}{a_{y x}}\right\}$ and satisfies the equation,

$$
\left(\frac{d_{y_{1}}}{a_{y x}}-x_{1}\right)\left(1-x_{1}\right)=\frac{a_{x y}}{a_{y x}} \frac{m_{21}\left(1-\varepsilon_{21}\right)}{r_{1}} y_{2}^{*} .
$$

It is easy to see that left-hand side of equation (2.3) is a concave-upward quadratic polynomial with roots 1 and $d_{y_{1}} / a_{y x}$ and intersects $y$-axis on the point $\left(0, d_{y_{1}} / a_{y x}\right)$. Hence if

$$
\frac{r_{2}}{r_{1}} \frac{a_{x y}}{b_{x y}}\left(m_{21}\left(1-\varepsilon_{21}\right)\right)\left(1-\frac{d_{y_{2}}+m_{21}}{b_{y x}}\right)<d_{y_{1}},
$$

then we can find an $x_{1}^{*}<\min \left\{1, \frac{d_{y_{1}}}{a_{y x}}\right\}$ satisfying (2.3). Next we can further discuss the local stabilities of all equilibrium of (2.1)-(2.2).

Direct computations, we can obtain the Jacobian matrix by direct computations,

$$
J=\left[\begin{array}{cccc}
J_{11} & -a_{x y} x_{1} & 0 & 0 \\
a_{y x} y_{1} & J_{22} & 0 & m_{21}\left(1-\varepsilon_{21}\right) \\
0 & 0 & J_{33} & -b_{x y} x_{2} \\
0 & 0 & b_{y x} y_{2} & J_{44}
\end{array}\right]
$$

where

$$
\begin{aligned}
& J_{11}=r_{1}\left(1-2 x_{1}\right)-a_{x y} y_{1}, \\
& J_{22}=-d_{y_{1}}+a_{y x} x_{1}, \\
& J_{33}=r_{2}\left(1-2 x_{2}\right)-b_{x y} y_{2}, \\
& J_{44}=-d_{y_{2}}+b_{y x} x_{2}-m_{21},
\end{aligned}
$$

Here we list all boundary equilibria and their corresponding local stabilities as follows.

(a) $E_{x_{1} x_{2}}$ : Evaluating the Jacobian matrix, we obtain

$$
J\left(E_{x_{1} x_{2}}\right)=\left[\begin{array}{cccc}
-r_{1} & -a_{x y} & 0 & 0 \\
0 & -d_{y_{1}}+a_{y x} & 0 & m_{21}\left(1-\varepsilon_{21}\right) \\
0 & 0 & -r_{2} & -b_{x y} \\
0 & 0 & 0 & -d_{y_{2}}-m_{21}+b_{y x}
\end{array}\right] .
$$

It is obvious that $E_{x_{1} x_{2}}$ is stable if $d_{y_{1}}>a_{y x}$ and $d_{y_{2}}+m_{21}>b_{y x}$. 
(b) $E_{1}$ : Similarly, evaluating the Jacobian matrix, we obtain

$$
J\left(E_{1}\right)=\left[\begin{array}{cccc}
-r_{1} x_{1}^{*}-a_{x y} x_{1}^{*} & 0 & 0 \\
a_{y x} y_{1}^{*} & 0 & 0 & m_{21}\left(1-\varepsilon_{21}\right) \\
0 & 0 & -r_{2} & -b_{x y} \\
0 & 0 & 0 & -d_{y_{2}}-m_{21}+b_{y x}
\end{array}\right],
$$

where $x_{1}^{*}=\frac{d_{y_{1}}}{a_{y x}}$ and $y_{1}^{*}=\frac{r_{1}}{a_{x y}}\left(1-x_{1}^{*}\right)$. It is easy to see that $E_{1}$ is stable if $d_{y_{2}}+m_{21}>b_{y x}$.

(c) $\bar{E}_{2}$ : Similarly, evaluating the Jacobian matrix, we obtain

$$
J\left(\bar{E}_{2}\right)=\left[\begin{array}{cccc}
r_{1}-a_{x y} y_{1}^{*} & 0 & 0 & 0 \\
a_{y x} y_{1}^{*} & -d_{y_{1}} & 0 & m_{21}\left(1-\varepsilon_{21}\right) \\
0 & 0 & -r_{2} x_{2}^{*} & -b_{x y} x_{2}^{*} \\
0 & 0 & b_{y x} y_{2}^{*} & 0
\end{array}\right],
$$

where $x_{2}^{*}=\frac{d_{y_{2}+m_{21}}}{b_{y x}}, y_{2}^{*}=\frac{r_{2}}{b_{x y}}\left(1-x_{2}^{*}\right)$, and $y_{1}^{*}=\frac{m_{21}\left(1-\varepsilon_{21}\right)}{d_{y_{1}}} y_{2}^{*}$. It is easy to see that $\bar{E}_{2}$ is stable if $r_{1}<a_{x y} y_{1}^{*}$ which is equivalent to

$$
\frac{r_{2}}{r_{1}} \frac{a_{x y}}{b_{x y}}\left(m_{21}\left(1-\varepsilon_{21}\right)\right)\left(1-\frac{d_{y_{2}}+m_{21}}{b_{y x}}\right)>d_{y_{1}} .
$$

(d) $\bar{E}_{*}$ : Evaluated the Jacobian matrix, we obtain

$$
J\left(\bar{E}_{*}\right)=\left[\begin{array}{cccc}
-r_{1} x_{1}^{*} & -a_{x y} x_{1}^{*} & 0 & 0 \\
a_{y x} y_{1}^{*}-d_{y_{1}}+a_{y x} x_{1}^{*} & 0 & m_{21}\left(1-\varepsilon_{21}\right) \\
0 & 0 & -r_{2} x_{2}^{*} & -b_{x y} x_{2}^{*} \\
0 & 0 & b_{y x} y_{2}^{*} & 0
\end{array}\right] .
$$

Since $x_{1}^{*}<d_{y_{1}} / a_{y x}$, it is clear that all eigenvalues of the Jacobian matrix $J\left(\bar{E}_{*}\right)$ are negative if $\bar{E}_{*}$ exists.

Therefore, we summarise the above arguments of local stabilities of equilibria in the following proposition.

Proposition 2.4. Consider the systems (2.1)-(2.2). The following statements are valid.

(i) The equilibrium $E_{x_{1} x_{2}}$ exists and is stable if $d_{y_{1}}>a_{y x}$ and $d_{y_{2}}+m_{21}>b_{y x}$.

(ii) The equilibrium $E_{1}$ exists if $a_{y x}>d_{y_{1}}$ and it is stable if $d_{y_{2}}+m_{21}>b_{y x}$.

(iii) The equilibrium $\bar{E}_{2}$ exists if $d_{y_{2}}+m_{21}<b_{y x}$ and it is stable if (2.6) holds.

(iv) The equilibrium $\bar{E}_{*}$ exists if $d_{y_{2}}+m_{21}<b_{y x}$ and (2.4) hold. Moreover, it is stable whenever it exists. 


\subsection{Classification and its corresponding global dynamics}

To clarify all global dynamics of (2.1) - (2.2) is not a easy task. Since these are four dimensional coupled systems with twelve parameters. How to take suitable parameters to classify is crucial. So we classify generically all parameters as six cases :

$\mathrm{I}: a_{y x}<d_{y_{1}}$ and $b_{y x}<d_{y_{2}}$;

II: $a_{y x}>d_{y_{1}}$ and $b_{y x}<d_{y_{2}}$;

III: $a_{y x}<d_{y_{1}}$ and $d_{y_{2}}<b_{y x}<d_{y_{2}}+m_{21}$;

IV: $a_{y x}>d_{y_{1}}$ and $d_{y_{2}}<b_{y x}<d_{y_{2}}+m_{21}$;

$\mathrm{V}: a_{y x}<d_{y_{1}}$ and $b_{y x}>d_{y_{2}}+m_{21}$

VI: $a_{y x}>d_{y_{1}}$ and $b_{y x}>d_{y_{2}}+m_{21}$.

The classification of parameters configuration are presented in Figure 2.1. First, we show a extinction result if the death rates of species $y_{1}$ and $y_{2}$ are over maximal resources which they can exploit. These parameters are showed in the case I of the Figure 2.1.

Proposition 2.5. If $d_{y_{1}}>a_{y x}$ and $d_{y_{2}}>b_{y x}$, then $\lim _{t \rightarrow \infty} y_{1}(t)=0$ and $\lim _{t \rightarrow \infty} y_{2}(t)=0$. Moreover, the boundary equilibrium $E_{x_{1} x_{2}}$ is globally asymptotically stable (GAS).

Proof. Let $\delta \equiv \frac{1}{2} \min \left\{d_{y_{1}}-a_{y x}, d_{y_{2}}-b_{y x}\right\}>0$. Since $\limsup \operatorname{sum}_{t \rightarrow \infty} x_{i}(t) \leq 1$, there is a positive time $T$ such that

$$
x_{1}(t)<1+\frac{\delta}{a_{y x}} \text { and } x_{2}(t)<1+\frac{\delta}{b_{y x}}
$$

for $t \geq T$. These will imply that $-d_{y_{1}}+a_{y x} x_{1}<-\delta$ and $-d_{y_{2}}+b_{y x} x_{2}<-\delta$ for $t \geq T$. Furthermore, we consider

$$
\begin{aligned}
\frac{\dot{y}_{1}+\dot{y}_{2}}{y_{1}+y_{2}} & =\frac{\left(-d_{y_{1}}+a_{y x} x_{1}\right) y_{1}}{y_{1}+y_{2}}+\frac{\left(-d_{y_{2}}+b_{y x} x_{2}\right) y_{2}}{y_{1}+y_{2}}-\frac{m_{12} \varepsilon_{12} y_{1}+m_{21} \varepsilon_{21} y_{2}}{y_{1}+y_{2}} \\
& \leq \frac{-\delta y_{1}}{y_{1}+y_{2}}+\frac{-\delta y_{2}}{y_{1}+y_{2}}=-\delta
\end{aligned}
$$

This implies that $\lim _{t \rightarrow \infty}\left(y_{1}(t)+y_{2}(t)\right)=0$. Because of positivity of $y_{1}(t)$ and $y_{2}(t)$, we obtain $\lim _{t \rightarrow \infty} y_{1}(t)=0$ and $\lim _{t \rightarrow \infty} y_{2}(t)=0$. We complete the proof.

Remark 2.6. This extinction result is still true if $m_{12}>0$. 


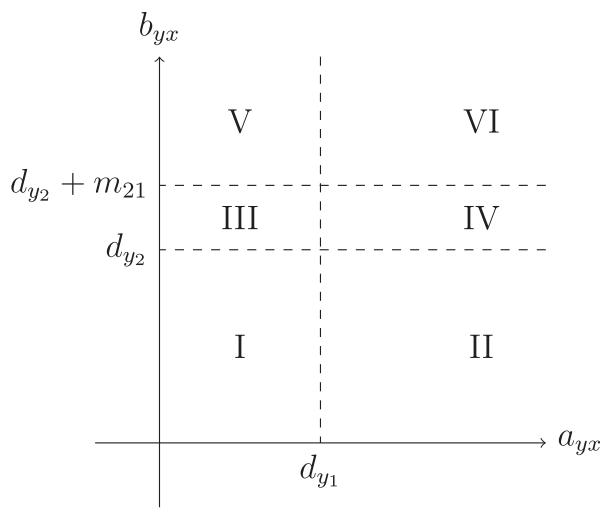

Figure 2.1: We classify the dynamics of (2.1) - (2.2) by parameters $a_{y x}$ and $b_{y x}$. Generically, we have six cases, case I : $a_{y x}<d_{y_{1}}$ and $b_{y x}<d_{y_{2}}$, case II $: a_{y x}>d_{y_{1}}$ and $b_{y x}<d_{y_{2}}$, case III $: a_{y x}<d_{y_{1}}$ and $d_{y_{2}}<b_{y x}<d_{y_{2}}+m_{21}$, case IV $: a_{y x}>d_{y_{1}}$ and $d_{y_{2}}<b_{y x}<d_{y_{2}}+m_{21}$, case V : $a_{y x}<d_{y_{1}}$ and $b_{y x}>d_{y_{2}}+m_{21}$, case VI $: a_{y x}>d_{y_{1}}$ and $b_{y x}>d_{y_{2}}+m_{21}$.

Proposition 2.7. Let assumption (A1) hold. If $b_{y x}<d_{y_{2}}+m_{21}$ then $\lim _{t \rightarrow \infty} y_{2}(t)=0$. If, in addition, $a_{y x}<d_{y_{1}}$, then $\lim _{t \rightarrow \infty} y_{1}(t)=0$ and the boundary equilibrium $E_{x_{1} x_{2}}$ is GAS. Otherwise, if, in addition, $a_{y x}>d_{y_{1}}$, then the boundary equilibrium $E_{1}=\left(\frac{d_{y_{1}}}{a_{x y}}, \frac{r_{1}}{a_{x y}}\left(1-\frac{d_{y_{1}}}{a_{x y}}\right), 1,0\right)$ is GAS.

Proof. It is clear to see that system (1.3) becomes the form,

$$
\begin{aligned}
& \frac{d x_{2}}{d t}=r_{2} x_{2}\left(1-x_{2}\right)-b_{x y} x_{2} y_{2}, \\
& \frac{d y_{2}}{d t}=\left(-d_{y_{2}}+b_{y x} x_{2}\right) y_{2}-m_{21} y_{2} .
\end{aligned}
$$

The differential inequality $\dot{y}_{2} / y_{2} \leq b_{y x}-d_{y_{2}}-m_{21}<0$ implies $\lim _{t \rightarrow \infty} y_{2}(t)=0$. Hence the remaining part of the proposition can be easily showed by the results of Proposition 2.1.

Remark 2.8. This result says that if parameters $\left(a_{y x}, b_{y x}\right)$ fall in region I-IV of Figure 2.1 then species $y_{2}$ will die out if $m_{12}=0$. Systems (2.1) -(2.2) become decoupled systems. Hence by results of Proposition 2.1, either $E_{x_{1} x_{2}}$ is GAS in region I, III or $E_{1}$ is GAS in region II, IV.

Before showing the GAS of equilibrium $\bar{E}_{2}$, we recall the following definitions and a theorem of Markus [5] which will be used here.

Definition. Let $A: \dot{x}=f(x, t)$ and $A_{\infty}: \dot{x}=f(x)$ be a first order system of ordinary differential equations. The real valued function $f(x, t)$ and $f(x)$ are continuous in $(x, t)$ for $x \in G$, where $G$ is an open subset of $\mathbb{R}^{n}$, and for $t>t_{0}$, and they satisfy a local Lipschitz condition in $x$. $A$ is said to be asymptotic to $A_{\infty},\left(A \rightarrow A_{\infty}\right)$ in $G$ if for each compact set $K \subset G$ and for each $\epsilon>0$, there is a $T=T(K, \epsilon)>t_{0}$ such that $|f(x, t)-f(x)|<\epsilon$ for all $x \in K$, and all $t>T$. 
Theorem (Markus). Let $A \rightarrow A_{\infty}$, in $G$ and let $P$ be an asymptotically stable critical point of $A_{\infty}$. Then there is a neighborhood $N$ of $P$ and a time $T$ such that the omega limit set for every solution $x(t)$ of $A$ which intersects $N$ at a time later than $T$ is equal to $P$.

Proposition 2.9. Let assumption (A1), $b_{y x}>d_{y_{2}}+m_{21}$ and (2.6) hold. Then the boundary equilibrium $\bar{E}_{2}=\left(0, y_{1}^{*}, x_{2}^{*}, y_{2}^{*}\right)$ of $(2.1)-(2.2)$ is GAS where $x_{2}^{*}=\frac{d_{y_{2}+m_{21}}}{b_{y x}}, y_{2}^{*}=\frac{r_{2}}{b_{x y}}\left(1-x_{2}^{*}\right)$, and $y_{1}^{*}=\frac{m_{21}\left(1-\varepsilon_{21}\right)}{d_{y_{1}}} y_{2}^{*}$.

Proof. Let $\left(x_{1}(t), y_{1}(t), x_{2}(t), y_{2}(t)\right)$ be a solution of (2.1)-(2.2) with positive initial conditions. First, we claim that $\left(x_{2}(t), y_{2}(t)\right)$ approaches $\left(x_{2}^{*}, y_{2}^{*}\right)$ as $t \rightarrow \infty$. Since it is obvious that (2.2) is decoupled with (2.1). So we consider the Lyapunov function

$$
L_{1}(t)=\int_{x_{2}(0)}^{x_{2}(t)} \frac{\eta-x_{2}^{*}}{\eta} d \eta+\frac{b_{x y}}{b_{y x}} \int_{y_{2}(0)}^{y_{2}(t)} \frac{\eta-y_{2}^{*}}{\eta} d \eta .
$$

Direct computation yields that

$$
\begin{aligned}
\frac{d L_{1}}{d t} & =\left(x_{2}-x_{2}^{*}\right) \frac{\dot{x}_{2}}{x_{2}}+\frac{b_{x y}}{b_{y x}}\left(y_{2}-y_{2}^{*}\right) \frac{\dot{y}_{2}}{y_{2}} \\
& =\left(x_{2}-x_{2}^{*}\right)\left(r_{2}\left(1-x_{2}\right)-b_{x y} y_{2}\right)+\frac{b_{x y}}{b_{y x}}\left(y_{2}-y_{2}^{*}\right)\left(-d_{y_{2}}-m_{21}+b_{y x} x_{2}\right) \\
& =-r_{2}\left(x_{2}-x_{2}^{*}\right)^{2} \leq 0 .
\end{aligned}
$$

Let $\mathscr{M} \equiv\left\{\left(x_{1}, y_{1}, x_{2}, y_{2}\right): \dot{L}_{1}\left(x_{1}, y_{1}, x_{2}, y_{2}\right)=0\right\}$ and it is obvious that $\mathscr{M}=\left\{\left(x_{1}, y_{1}, x_{2}^{*}, y_{2}\right): x_{1}>\right.$ $\left.0, y_{1}>0, y_{2}>0\right\}$ with maximal invariant subset of $\mathscr{M},\left\{\left(x_{1}, y_{1}, x_{2}^{*}, y_{2}^{*}\right): x_{1}>0, y_{1}>0\right\}$. Hence, by LaSalle's invariant principle, we prove the claim, $\left(x_{2}(t), y_{2}(t)\right) \rightarrow\left(x_{2}^{*}, y_{2}^{*}\right)$ as $t \rightarrow \infty$.

Applying the Markus theorem to

$$
A:\left\{\begin{array}{l}
\dot{x}_{1}=r_{1} x_{1}\left(1-x_{1}\right)-a_{x y} x_{1} y_{1} \\
\dot{y}_{1}=\left(-d_{y_{1}}+a_{y x} x_{1}\right) y_{1}+m_{21}\left(1-\varepsilon_{21}\right) y_{2} \\
\dot{x}_{2}=r_{2} x_{2}\left(1-x_{2}\right)-b_{x y} x_{2} y_{2} \\
\dot{y}_{2}=\left(-d_{y_{2}}+b_{y x} x_{2}\right) y_{2}-m_{21} y_{2} \\
\quad x_{i}(0)>0, y_{i}(0)>0
\end{array}\right.
$$

and

$$
A_{\infty}:\left\{\begin{array}{l}
\dot{x}_{1}=r_{1} x_{1}\left(1-x_{1}\right)-a_{x y} x_{1} y_{1}, \\
\dot{y}_{1}=\left(-d_{y_{1}}+a_{y x} x_{1}\right) y_{1}+m_{21}\left(1-\varepsilon_{21}\right) y_{2}^{*}, \\
\dot{x}_{2}=0, \\
\dot{y}_{2}=0, \\
\quad x_{1}(0)>0, y_{1}(0)>0, x_{2}(0)=x_{2}^{*}, y_{2}(0)=y_{2}^{*},
\end{array}\right.
$$


Table 2.1: Parmeters to simulate the global dynamics of (2.1)-(2.2) numerically.

\begin{tabular}{|c|c|c|c|c|c|c|c|c|c|}
\hline$r_{1}$ & $a_{x y}$ & $a_{y x}$ & $d_{y_{1}}$ & $r_{2}$ & $b_{x y}$ & $b_{y x}$ & $d_{y_{2}}$ & $m_{21}$ & $\varepsilon_{21}$ \\
\hline 0.6 & 1 & 1 & 0.1 & 0.6 & 0.5 & 1 & 0.4 & 0.4 & 0.1 \\
\hline 0.6 & 1 & 1 & 0.5 & 0.6 & 0.5 & 1 & 0.4 & 0.4 & 0.1 \\
\hline
\end{tabular}

it follows that solutions of (2.7) approach $\bar{E}_{2}$ if we can show that solutions of (2.8) approach $\bar{E}_{2}$.

It is sufficient to complete the proof if we can show that $\left(x_{1}(t), y_{1}(t)\right)$ of the solution of (2.8) approaches $\left(0, y_{1}^{*}\right)$. Now we define another Lyapunov function for (2.8),

$$
L_{2}(t)=x_{1}(t)+\frac{a_{x y}}{a_{y x}} \int_{y_{1}(0)}^{y_{1}(t)} \frac{\eta-y_{1}^{*}}{\eta} d \eta .
$$

By direct computations, we have

$$
\begin{aligned}
\frac{d L_{2}}{d t}= & r_{1} x_{1}\left(1-x_{1}\right)-a_{x y} x_{1} y_{1}+\frac{a_{x y}}{a_{y x}}\left(y_{1}-y_{1}^{*}\right)\left[-d_{y_{1}}+a_{y x} x_{1}+m_{21}\left(1-\varepsilon_{21}\right) \frac{y_{2}^{*}}{y_{1}}\right] \\
= & r_{1} x_{1}\left(1-x_{1}\right)-a_{x y} x_{1} y_{1}+a_{x y} x_{1}\left(y_{1}-y_{1}^{*}\right)+ \\
& \frac{a_{x y}}{a_{y x}}\left(y_{1}-y_{1}^{*}\right)\left[-m_{21}\left(1-\varepsilon_{21}\right) \frac{y_{2}^{*}}{y_{1}^{*}}+m_{21}\left(1-\varepsilon_{21}\right) \frac{y_{2}^{*}}{y_{1}}\right] \\
= & r_{1} x_{1}\left(1-x_{1}\right)-a_{x y} x_{1} y_{1}^{*}+\frac{a_{x y}}{a_{y x}}\left(y_{1}-y_{1}^{*}\right) m_{21}\left(1-\varepsilon_{21}\right)\left(\frac{y_{2}^{*}}{y_{1}}-\frac{y_{2}^{*}}{y_{1}^{*}}\right) \\
= & -r_{1} x_{1}^{2}-x_{1}\left(a_{x y} y_{1}^{*}-r_{1}\right)-\frac{a_{x y}}{a_{y x}} m_{21}\left(1-\varepsilon_{21}\right) \frac{y_{2}^{*}\left(y_{1}-y_{1}^{*}\right)^{2}}{y_{1} y_{1}^{*}} \leq 0,
\end{aligned}
$$

where note that (2.6) is equivalent to $r_{1}<a_{x y} y_{1}^{*}$. Similarly, by LaSalle's invariant principle, $\left(x_{1}(t), y_{1}(t)\right)$ of the solution of (2.8) approaches $\left(0, y_{1}^{*}\right)$. We complete the proof.

Finally, we would like to present the global dynamics of (2.1)-(2.2) with parameters $\left(a_{y x}\right.$, $\left.b_{y x}\right)$ falling in region V and VI of Figure 1 numerically, that is, we ask that $b_{y x}>d_{y_{2}}+m_{21}$ and take parameters in following table. By direct computations, we have

$$
\frac{r_{2}}{r_{1}} \frac{a_{x y}}{b_{x y}} m_{21}\left(1-\varepsilon_{21}\right)\left(1-\frac{d_{y_{2}}+m_{21}}{b_{y x}}\right)=0.144 .
$$

If we take parameters in the first row of Table 2.1 with $d_{y_{1}}=0.1$, then (2.6) holds which implies $\bar{E}_{2}$ is globally asymptotically stable by Proposition 2.9. Please refer Figure 2.2. Similarly, we take parameters in the second row of Table 2.1 with $d_{y_{1}}=0.5$, then (2.4) holds which implies $\bar{E}_{*}$ exists and is stable by Proposition 2.4. Numerically, we see these facts in Figure 2.3. Finally, we conjecture that the positive equilibrium $\bar{E}_{*}$ is globally asymptotically stable whenever is exists. 


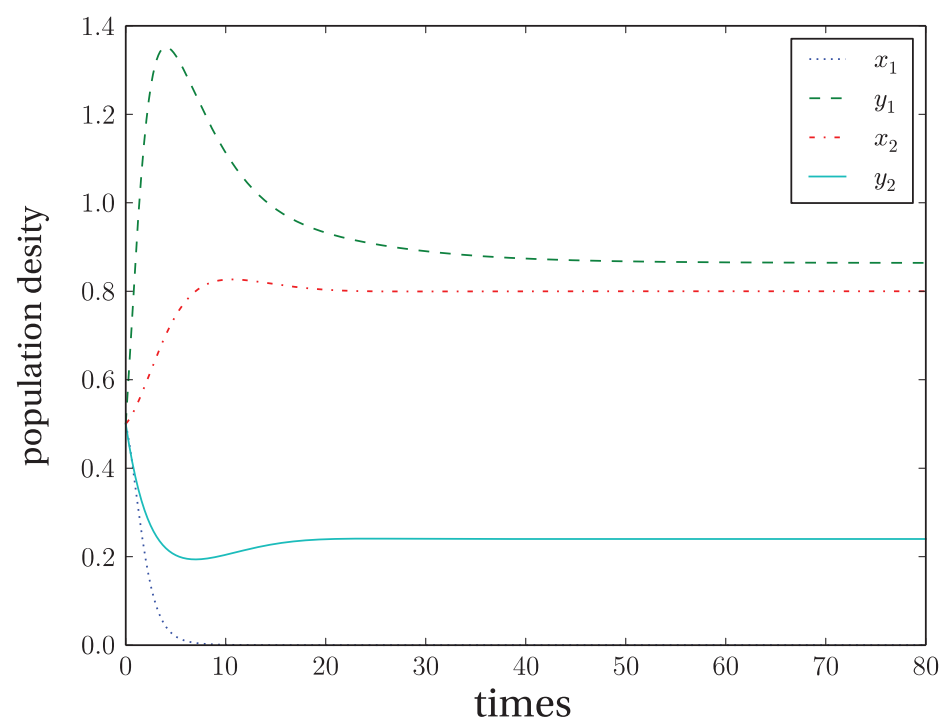

Figure 2.2: Time courses of numerical simulation of each species of (2.1)-(2.2) with parameters and $d_{y_{1}}=0.1$ in Table 2.1 .

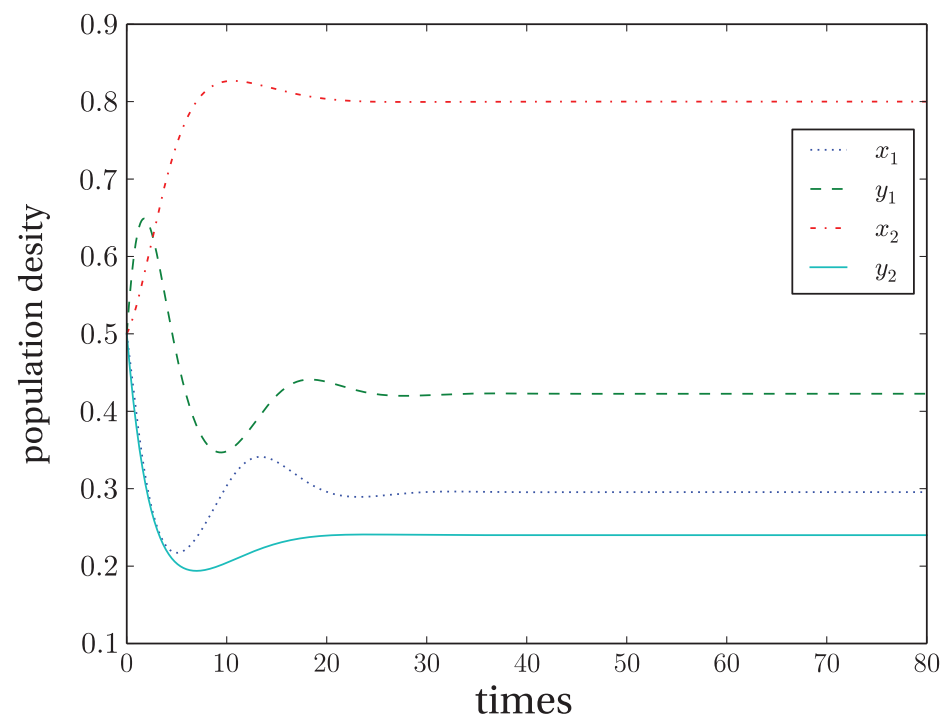

Figure 2.3: Time courses of numerical simulation of each species of (2.1)-(2.2) with parameters and $d_{y_{1}}=0.5$ in Table 2.1 .

\section{Discussions and biological interpretations}

In this work, we consider the dynamics of predator-prey systems on a fragmented habitat with two patches. Each patch contains a two-species predator-prey ecological system. Moreover the predator species can disperse between the two patches with dispersal rates $m_{12}\left(m_{21}\right)$ 
and dispersal costs $\varepsilon_{12}\left(\varepsilon_{21}\right)$. To simplify the arguments, we further assume that the dispersal is asymmetric, i.e. $0=m_{12}<m_{21}$. Analytically, we find conditions guaranteeing the existence of boundary and interior equilibria. Moreover, local stability and global extinctions of all equilibria are established using differential inequalities and LaSalle's invariance principle.

It is well-known that for a predator-prey system on one patch with strong Allee effect in the prey, a large predator invasion leads to the extinction of both species. Mathematically it is called bi-stability whereas in biology it is called over-exploitation by the predator [10, 11, 12] It is interesting that even though each of the predator-prey systems (1.2)-(1.3) has no Allee effect in the prey, we have been able to show in Proposition 2.9 that the boundary equilibrium $E_{2}$ is globally asymptotically stable if the inequality below holds,

$$
\frac{r_{2}}{r_{1}} \frac{a_{x y}}{b_{x y}}\left(m_{21}\left(1-\varepsilon_{21}\right)\right)\left(1-\frac{d_{y_{2}}+m_{21}}{b_{y x}}\right)>d_{y_{1}} \text {, or equivalently } r_{1}<a_{x y} y_{1}^{*} .
$$

Let us carefully re-examine this inequality. It will hold if,

[1] the predator of patch 2 survives $\left(d_{y_{2}}+m_{21}<b_{y x}\right)$;

[2] the dispersal rate $\left(m_{21}\right)$ is larger and the dispersal cost $\left(\varepsilon_{21}\right)$ is smaller;

[3] the resource in patch 1 cannot sustain the exploitation by the consumer in patch $1\left(r_{1}<\right.$ $\left.a_{x y} y_{1}^{*}\right)$.

Up to now the phenomenon of overexploitation by predator has only been found in the predatorprey model with strong Allee effect in the prey in the ODE case [10, 12] or PDE case [11]. In Proposition 2.9 we show a similar phenomenon arises when the resource in patch 1 cannot sustain the exploitation by the consumer in patch 1 . There is no Allee effect in systems (1.2)-(1.3). Hence we suggest that the reason is due to the fragmentation of habitat.

For the case $m_{12}>0$, the analysis will be another long story. So we leave it as another project. Here we only present an interesting numerical result.Take the same parameters as in the first row of Table 2.1. By the previous results, if $m_{12}=0$, then we have $\lim _{t \rightarrow \infty} x_{1}(t)=0$, i.e. the species $x_{1}$ will die out eventually. Mathematically, the numerical results suggest that the positive equilibrium does not exist. However, we take $m_{12}>0$ and fix $m_{12} \in[0.1,0.2]$ with step size 0.001 . For each fixed parameter $m_{12}$, we calculate the asymptotic state of (1.2)-(1.3) numerically. We find that if $m_{12}$ is large enough( $\left.>0.15\right)$, then the species $x_{1}$ will survive and a positive equilibrium $\bar{E}_{*}$ exists. Moreover, the asymptotic density of species $x_{1}$ increases with respect to parameter $m_{12}$. Please refer to Figure 3.4. Numerically, it is obvious that the dispersal affects the global dynamics positively. Moreover, the numerical results also suggest that the habitat corridor which increases the rate of movement enhances biodiversity. 


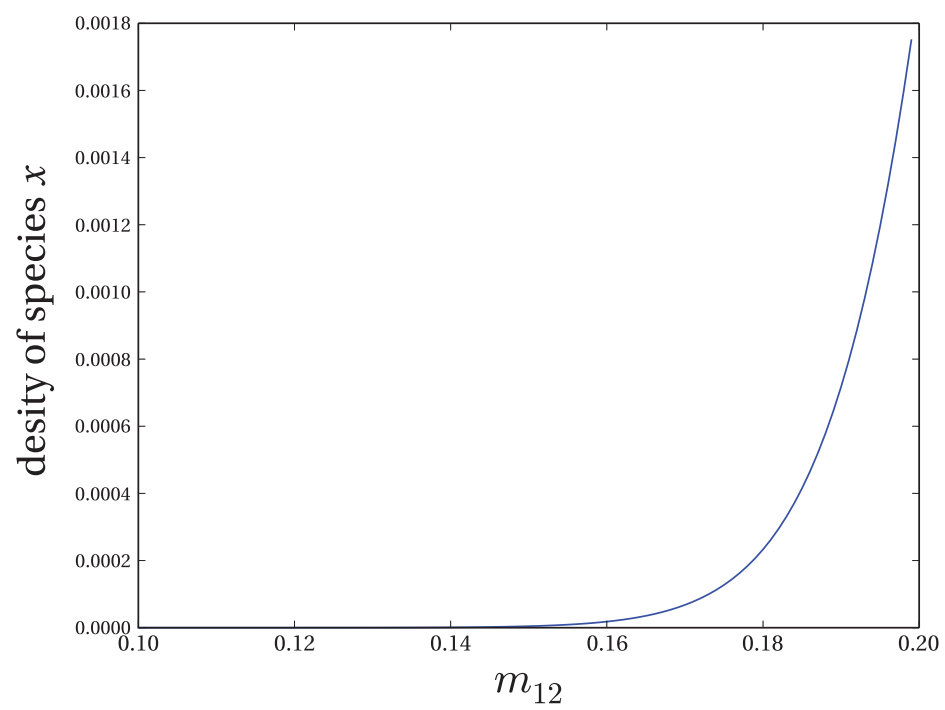

Figure 3.4: Numerical simulations of the asymptotic state of species $x_{1}$ of (1.2)-(1.3) for values of parameter $m_{12}$ from 0.1 to 0.2 with step size 0.001 ; the other parameters are the same as in the first row of Table 2.1.

\section{Acknowledgement}

Research of this paper was partially performed when the corresponding author (T.-H. Yang) was visiting the National Center for Theoretical Sciences (NCTS), Hsinchu, the kind hospitality and professional assistance of the staff and members of NCTS is greatly appreciated.

\section{References}

[1] R. Cressman and V. Křivan, Two-patch population models with adaptive dispersal: the effects of varying dispersal speeds, Journal of Mathematical Biology, 67 (2013), 329-358.

[2] D. L. DeAngelis, G. S. K. Wolkowicz, Y. Lou, Y. Jiang, M. Novak, R. Svanbäck, M. S. Araújo, Y. Jo and E. A. Cleary, The effect of travel loss on evolutionarily stable distributions of populations in space, The American naturalist, 178 (2011), 15-29.

[3] R. Levins, Evolution in Changing Environments. Princeton University Press, Princeton, 1969.

[4] Y. Lou and C.-H. Wu, Global dynamics of a tritrophic model for two patches with cost of dispersal, SIAM Journal on Applied Mathematics, 71(2011), 1801-1820.

[5] L. Markus, Asymptotically autonomous differential systems, In Contributions to the theory of nonlinear oscillations, vol. 3, 17-29. Princeton University Press, Princeton, N. J., 1956.

[6] M. L. McKinney, Urbanization, Biodiversity, and Conservation The impacts of urbanization on native species are poorly studied, but educating a highly urbanized human population about these impacts can greatly improve species conservation in all ecosystems, BioScience, 52 (2002), 883-890.

[7] T. Namba, A. Umemoto and E. Minami, The effects of habitat fragmentation on persistence of source-sink metapopulations in systems with predators and prey or apparent competitors, Theoretical Population Biology, 56 (1999), 123-137. 
[8] M. R. W. Rands, W. M. Adams, L. Bennun, S. H. M. Butchart, A. Clements, D. Coomes, A. Entwistle, I. Hodge, V. Kapos, J. P. W. Scharlemann, W. J. Sutherland and B. Vira. Biodiversity Conservation: Challenges Beyond 2010, Science (New York, NY), 329 (201), 1298-1303.

[9] S. P. D. Riley, R. M. Sauvajot, T. K. Fuller, E. C. York, D. A. Kamradt, C. Bromley and R. K. Wayne, Effects of Urbanization and Habitat Fragmentation on Bobcats and Coyotes in Southern California, Conservation Biology, 17 (2003), 566-576.

[10] G. A. K. van Voorn, L. Hemerik, M. P. Boer and B. W. Kooi, Heteroclinic orbits indicate overexploitation in predator-prey systems with a strong Allee effect, Mathematical Biosciences, 209(2007), 451-469.

[11] J. Wang, J. Shi and J. Wei. Dynamics and pattern formation in a diffusive predator-prey system with strong Allee effect in prey, J Differential Equations, 251 (2011), 1276-1304.

[12] J. Wang, J. Shi and J. Wei, Predator-prey system with strong Allee effect in prey. Journal of Mathematical Biology, 62 (2011), 291-331.

Department of Mathematics, Tamkang University, Tamsui, New Taipei City 25137, Taiwan.

E-mail: ckj25_1999@yahoo.com.tw

E-mail: thyang@mail.tku.edu.tw

E-mail: happyeat0625@yahoo.com.tw 